\title{
An Analysis of Broad Emission-Line Profiles from HST Data
}

\author{
Stephanie A. Snedden and C. Martin Gaskell \\ Physics and Astronomy Department, University of Nebraska, Lincoln, \\ NE 68588-0111, USA
}

\begin{abstract}
We have analyzed the $H S T$ FOS spectra of all quasars in the Stirpe (1990) high $S / N$ line-profile sample and studied line-profile ratios as a function of radial velocity. Some quasars show no sign at all of NLR Ly $\alpha$. We confirm that $\mathrm{H} \alpha$ is narrower than Ly $\alpha$ (after allowance for NLR contributions). The $\mathrm{Ly} \alpha / \mathrm{H} \alpha$ ratios in the cores of the broad lines are all close to or slightly less than case B and values predicted by single-cloud photoionization models. The Ly $\alpha / \mathrm{H} \alpha$ ratio is surprisingly high in the blue wing. With only one exception, the ratios are equal to or greater than the case $B$ value. Intrinsic reddening must be very small in most cases. We also briefly discuss other ratios.
\end{abstract}

\section{Introduction}

There are many reasons for believing that the low-ionization BLR lines come from quite different clouds than the high-ionization lines. There are difficulties in explaining all the lines from a single type of cloud: the high-ionization lines are blueshifted relative to the low-ionization lines, the high-ionization lines have broader profiles, and variability studies show that the low- and high-ionization lines come from slightly different radii (see Gaskell 1987). Line variability transfer functions also probably imply that the two regions have different structure (e.g., Horne, Welsh, \& Peterson 1991).

We present here a preliminary study of line ratios as a function of velocity for eight quasars with both optical and HST FOS spectra. Stirpe (1990) has published high-quality optical spectra of a number of quasars. We have selected those with high $S / N$ FOS spectra.

\section{Results}

The ratio of two lines of different widths vs. velocity will clearly show a curvature that can be approximated with a quadratic. The Ly $\alpha / \mathrm{H} \alpha$ plots have curvature in the sense that confirms that $\mathrm{H} \alpha$ is narrower than Ly $\alpha$ (Zheng 1992; Netzer et al. 1995). Interestingly, in two of the quasars there is no sign of a NLR Ly $\alpha$ contribution (see Fig. 1; note that narrow-line components were removed from $\mathrm{H} \alpha$ but not from Ly $\alpha$ ). This can easily be explained by known NLR reddening (Wysota \& Gaskell 1988). 

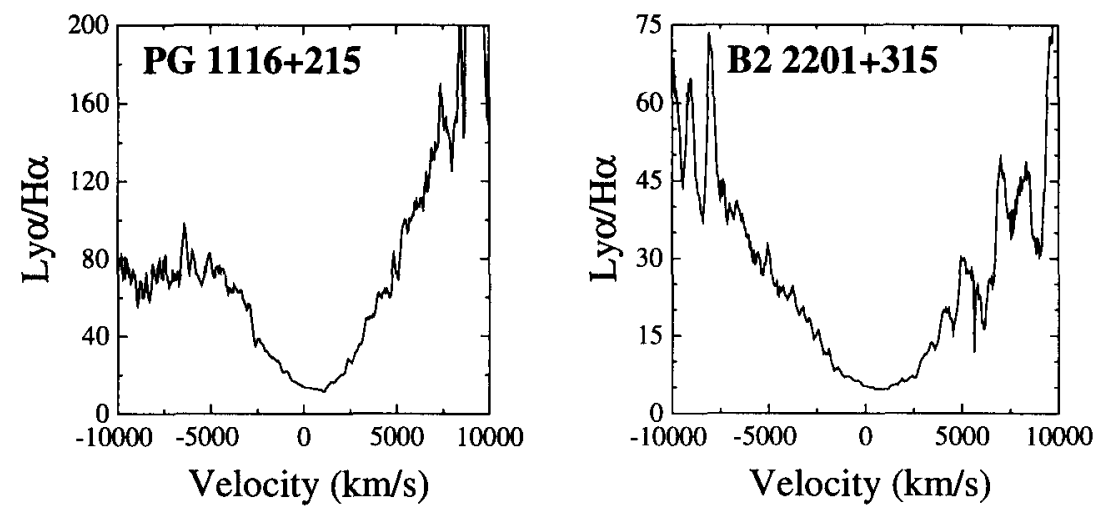

Figure 1. Ly $\alpha / \mathrm{H} \alpha$ profile ratios for $\mathrm{PG} 1116+215$ and $\mathrm{B} 22201+315$.

With one exception, the $\mathrm{Ly} \alpha / \mathrm{H} \alpha$ ratios in the blue wing equal or exceed the case $B$ value of 8.7 (Ferland 1995). The red wing ratio also appears high, although there are contamination problems due to $\mathrm{N} \mathrm{V}$. The high Ly $\alpha / \mathrm{H} \alpha$ ratio in the blue wing is surprising. At the very least it implies there is essentially no reddening along the line of sight to these quasars. Since there is probably some reddening from our Galaxy, the intrinsic Ly $\alpha / \mathrm{H} \alpha$ ratios might be greater than case $B$.

On the other hand, in the cores of the broad lines, the values of $\mathrm{Ly} \alpha / \mathrm{H} \alpha$ are $\lesssim 8.7$, in agreement with photoionization models (e.g., Ferland 1995).

The ionization-parameter-sensitive and density-sensitive $\mathrm{CIII}$ / C IV ratio shows a variety of dependences with velocity. However, the VBLR, ILR, and NLR have the same C III]/C IV ratios in this sample. The Ly $\alpha / C$ IV ratio is fairly constant as a function of velocity, especially when narrow Ly $\alpha$ is absent.

Acknowledgments. This work has been supported in part by grant AR05796.01-94A from the Space Telescope Science Institute, which is operated by AURA, Inc., under NASA contract NAS5-26555.

\section{References}

Ferland, G.J. 1995, Univ. Kentucky Internal Report.

Gaskell, C. M. 1987, in Astrophysical Jets \& their Engines, ed. W. Kundt (Reidel: Dordecht), p. 29.

Horne, K., Welsh, W. F., \& Peterson, B. M. 1991, ApJ, 367, L5.

Netzer, H., Brotherton, M.S., Wills, B.J., Han, M., Wills, D., Baldwin, J.A., Ferland, G.J., \& Browne, I. W. A. 1995, ApJ, 448, 27.

Stirpe, G. M. 1990, A\&AS, 85, 1049.

Wysota, A., \& Gaskell, C.M. 1988, in Active Galactic Nuclei, eds. H. R. Miller \& P.J. Wiita, (Springer: Berlin), p. 79.

Zheng, W. 1992, ApJ, 385, 127. 